\title{
Multilayer Learning Network for Modulation Classification Assisted with Frequency Offset Cancellation in Satellite to Ground Link
}

\author{
Guan Qing Yang $\mathbb{1}$ \\ College of Electronic and Information Engineering, Shenyang Aerospace University, Shenyang 110136, China \\ Correspondence should be addressed to Guan Qing Yang; guan_qy@163.com
}

Received 18 September 2017; Revised 27 February 2018; Accepted 31 March 2018; Published 10 May 2018

Academic Editor: Ioannis Krikidis

Copyright (C) 2018 Guan Qing Yang. This is an open access article distributed under the Creative Commons Attribution License, which permits unrestricted use, distribution, and reproduction in any medium, provided the original work is properly cited.

\begin{abstract}
A multilayer learning network assisted with frequency offset cancellation is proposed for modulation classification in satellite to ground link. Carrier frequency offset greatly reduces modulation classification performance. It is necessary to cancel frequency offset before modulation classification. Frequency offset cancellation weights are established through multilayer learning network based on MSE criterion. Then the weight and hidden layer of multilayer learning network are also established for modulation classification. The hidden layers and weight are trained and tuned to combat the interference introduced by frequency offset. Compared with current modulation classification algorithm, the proposed multilayer learning network greatly improves the Probability of Correct Classification (PCC). It has been proven that the proposed multilayer learning network assisted with frequency offset has higher performance for modulation classification within the same training sequence.
\end{abstract}

\section{Introduction}

Satellite to ground link adopts different modulation technology to satisfy different requirement. With rapid development of communication technology, modulation classification for satellite communication becomes an important research topic in signal recognition, especially in lager frequency offset environment [1-3].

Doppler frequency offset exists between users and satellite, and it greatly damages the link performance and especially affects modulation classification. As satellite is moving at high speed, frequency offsets are also changing. This process requires that modulation classification should overcome frequency offsets within larger SNR dynamic range. So it is very important to adopt frequency offset cancellation method for modulation classification.

In early research work, the literatures were researching on cancellation algorithms for frequency offsets, which were estimated at receivers and then sent back to respective transmitter. But this is not suitable for long-distance transmission in satellite to ground link. Reference [4] analyzed single user in earliest frequency offset cancellation algorithm. Least squares (LS) and minimum mean-square error (MMSE) were applied in [5]. LS method required frequency offset, while MMSE algorithm required noise powers as well. Reference [6] proposed an iterative cancellation for canceling frequency offset interference, which should need large complex iterations. Huang gave frequency offsets cancellation method using circular convolution in [7], which should need relatively large complexity.

Reference [8] proposed an alternative method in timedomain method for frequency offset compensation, but the precision was not very satisfactory. Reference [9] proposed a joint iterative detection algorithm, which should require ICI matrix inversion in each iteration. In [10], successive interference cancellation (SIC) algorithm was proposed to cancel frequency offset interference. The interference was cancelled by reconstructing signals. SIC algorithm depending on power order was proposed in [11]. Reference [12] gave SIC algorithm based on the signal-to-interference-plus-noise ratio (SINR) order. Reference [13] proposed an iterative parallel interference canceller (PIC) solution. Based on this, selective parallel interference cancellation (SPIC) algorithm was presented in [14] but had a significant implementation 
complexity. Based on signal-to-interference ratio (SIR) analyzing, a parallel interference canceller for mitigation of interference due to CFOs was presented in [15].

Modulation classification for communication signal in satellite link is the multivariate pattern classification problem with multiple unknown parameters. Modulation classification algorithms should extract the feature from sampling signal. These algorithms can be classified into the following 5 types.

Type 1 Histogram Features. In 1984, Liedtke et al. proposed a pattern recognition method to classify digital modulated signals. Liedtke et al. used amplitude histogram, frequency histogram, and phase histogram as characteristic parameters. Too many feature dimensions lead to lager computational complexity. However, reducing feature dimensions would affect the ability of different modulation classification with similar distribution functions.

Type 2 Statistical Cumulant Features. In view of the larger number of histogram features, literature [16] proposed a sixth-order cumulants feature in order to improve the performance. However, the sixth-order cumulants algorithm was only based on the energy model, which was not effective for frequency offset. In [17-19], cyclic high-order cumulants were obtained for modulation classification, and the performance in the Gaussian white noise channel was given. But the performance in large frequency offset status was not considered. References [20,21] used high-order cumulant feature for modulation classification. These feature extraction operations were relatively easy to obtain. However, computational complexity for higher-order cumulants was improved. As modulation classification algorithms obtained features based on cumulants, classification performance within larger frequency offset was not in consideration.

Asoke K. Nandi and EEAzzouz et al. used the statistical features of instantaneous envelope, phase, and frequency of signal data and applied decision theory for modulation classification [22, 23]. A similar algorithm was proposed by Chan and Gadbois. According to the features of the signal envelope, the ratio of the signal envelope variance to the signal mean square was used as the decision criterion [24]. Louis used digital signals such as 2FSK, 4FSK, 8FSK, OQPSK, MSK, BPSK, QPSK, 8PSK, 16QAM, 64QAM, and other digital signals based on instantaneous frequency, phase, and amplitude [17]. Swami adopted normalized cumulative power of the symbol-synchronous sampling sequence to classify QAM, PSK, ASK, and other signals [20]. In 2001, Wong and Nandi [25] used statistical cumulant and spectral features in classification.

Type 3 Transformation Feature Criterion. Cumulant features for satellite signal are only to identify several common modulations. In addition to directly using the statistical parameters and the histogram as a classification feature, the signal can be transformed into other feature spaces and the satellite data analysis can be accomplished by using the feature parameters in the new feature space. With the development of signal processing theory, the signal could be transformed into various forms. Wavelet transformation feature for modulation classification was proposed in [26]. Haar wavelet feature transform had better antinoise features than the high-order cumulants. But it had weak anti-frequency offset features. In [27], modulation classification algorithm based on cycle spectrum features was proposed. Based on extending this algorithm, modulation classification algorithm based on bispectrum feature was used to classify MPSK signal in [28]. In [29], wavelet packet was proposed, which was decomposed by the wavelet packet. The partial decomposition vector of the average energy was obtained. Then average energy was arranged in a certain order to construct classification feature. In [30], MFSK and MPSK are classified through wavelet transform, and better PCC was obtained when the SNR was greater than $6 \mathrm{~dB}$. In [31], an automatic classification algorithm based on spectral analysis was proposed, which used the statistical characteristics assisted with maximum likelihood estimator.

Type 4 Bayesian Classification Criterion. A classical method for modulation classification is the maximum likelihood method. Modulation classification is obtained through maximum likelihood function. In [32], MPSK signal based on phase maximum likelihood function was proposed. In [33], a joint likelihood function using amplitude and phase was proposed within larger SNR, and it was on the premise that the amplitude and phase were assumed to be independent. In [34], classification for MFSK signal was discussed. After averaging the unknown parameters in the average likelihood function, the integral expression of zero-order modified Bessel function was obtained. The high-order correlation analysis based on Bessel function was derived for modulation classification. In literature [35], a generalized likelihood ratio function for classification framework was proposed, which firstly expanded the power for the likelihood ratio function and then made the expected average processing for the unknown parameters and then formed the classification statistics based on higher correlation order.

The advantage of transformation domain theory for modulation classification is theoretically guaranteeing that the result is optimal under Bayesian cost criterion. The classification performance curve can be obtained by theoretical analysis. The likelihood ratio performance can be used as a theoretical upper limitation. Of course, the maximum likelihood algorithm also has limitations and shortcomings. Firstly, compared to the modulation classification method, it requires more a priori knowledge, such as the form of the distribution function including the mean, variance, and signal-to-noise ratio. Secondly, the existence of unknown parameters results in the complex calculation, which is also difficult for real-time processing.

In order to cancel frequency offset, the eigenvalue is very limited to suppress the noise based on Bayesian classification criterion. Current methods such as feature extraction, classification, and regression can be used as shallow structure algorithms. These methods are limited to complex functions in finite samples and computational units, and their generalization ability is restricted to complex classification problems. 


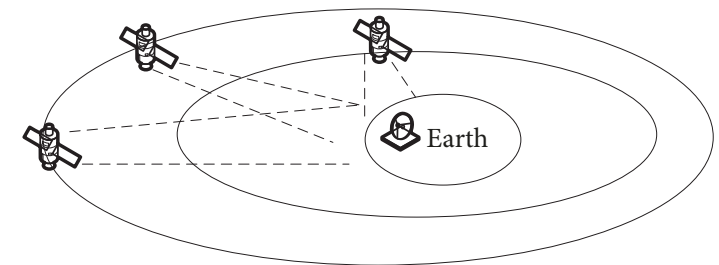

FIGURE 1: Satellite beam enters the target ground station antenna range.

Type 5 Multilayer Neural Network. With improvement of artificial intelligence, researchers introduced multilayer neural network algorithms for modulation classification. Multilayer learning could represent the input data distribution by learning a multilayer nonlinear network structure and implementing complex function approximation. The multilayer learning motivation lies in the establishment of a neural network, which could simulate the human brain for signal analysis and mimic human brain mechanisms to interpret the data.

Multilayer learning algorithms obtain data features by building models with multiple hidden layers to improve classification or prediction accuracy. Multilayer learning algorithm is different from the traditional shallow learning structure; the difference in multilayer learning lies in the following: (1) emphasizing the depth of the model structure usually with many hidden nodes and (2) the importance of feature learning being clearly highlighted. By layer-by-layer feature transformation, the feature of data in the original space is transformed into a new feature space to make classification or prediction easier. Compared with the method of constructing data features manually, the use of data to learn features can better express intrinsic information. Identification part needs to determine the appropriate decision rules and classifier structure.

Common classifiers include tree structure classifiers [36, 37], neural network classifiers [38, 39], and support vector machine classifiers $[40,41]$. The tree structure classifier used a multilevel classifier structure, each structure according to one or more of the characteristic parameters. Neural network classifier uses different structures of artificial neural network for a variety of ways to achieve training and testing. SVM classifier inputs feature vector into highdimensional space and constructs the optimal classification in the high-dimensional space to achieve data classification. In [42], a classifier based on tree structure was adopted. The multilevel tree classifier structure is based on one or more feature parameters. In [43], a multilayer clustering algorithm is used for modulation classification. In [44], $K$ nearest neighbor algorithm classifier was used to identify modulation mode. Literature [45] used the distance distribution function to optimize and obtain better classification performance. However, this algorithm did not give analysis in larger frequency offset. In [46], an improved KNN multilayer learning architecture was proposed to classify modulation. However, this algorithm was applicable for Gaussian white noise channel environment, and the performance of the algorithm was limited for larger frequency offset.

\section{System Model and Problem Formulation}

2.1. Satellite to Ground Link Model. Due to high-speed movement of the satellite, the satellite to ground link is established between station and satellite. In this process, it is necessary to accurately calculate the constellation. The process has been shown in Figure 1. In this process, frequency offset of the satellite to ground link is changing, so it is necessary to accurately cancel the interference of frequency offset before modulation classification.

We establish the satellite to ground link communication model. Ossanna has firstly proposed the probability statistical model of satellite to ground link in 1964 [47]. The principle is based on the reflection wave interference, so the angle of reflection is limited. It is fuzzy to use this satellite ground to link model to describe the rural and urban areas. Clarke proposed in 1968 statistical model [48] based on the principle of scattering signal strength, but the model needed to assume that the incident wave formation was the same, so the application of the model was limited. After the 70s, according to the measured data in Canada, Loo proposed a statistical model based on [49]. If the shadow fading follows the Nakagami distribution, the Abdi star fading model is formed [50]. So, it is common for satellite to use probability density function, such as Rician model, Loo model, and Rician-Lognormal model. The scene for the satellite to ground communication system is mainly urban environment, and most of signal transmission has direct path.

2.2. Signal Processing Model. The $n$th received frame can be written as

$$
x(n)=d(n) * h(n), \quad n=1, \ldots, N,
$$

where $h(n)$ represents channel impulse response at instant $n$. At the receiver, frequency offset is induced into the baseband signal due to Doppler frequency and the clock oscillator misalignment between the satellite and user. The baseband signal could be given as

$$
s(n)=x(n) e^{j 2 \pi n \xi / N}+z(n), \quad n=1, \ldots, N
$$

where $\xi$ denotes frequency offset (FO) normalized by the subcarrier spacing and $z(n)$ is the additive white Gaussian noise with zero mean and $\sigma^{2}$ variance.

As shown in Figure 2, the multipath fading satellite communication signal should pass through the downconversion sampling block. Signal-to-noise ratio should be estimated 


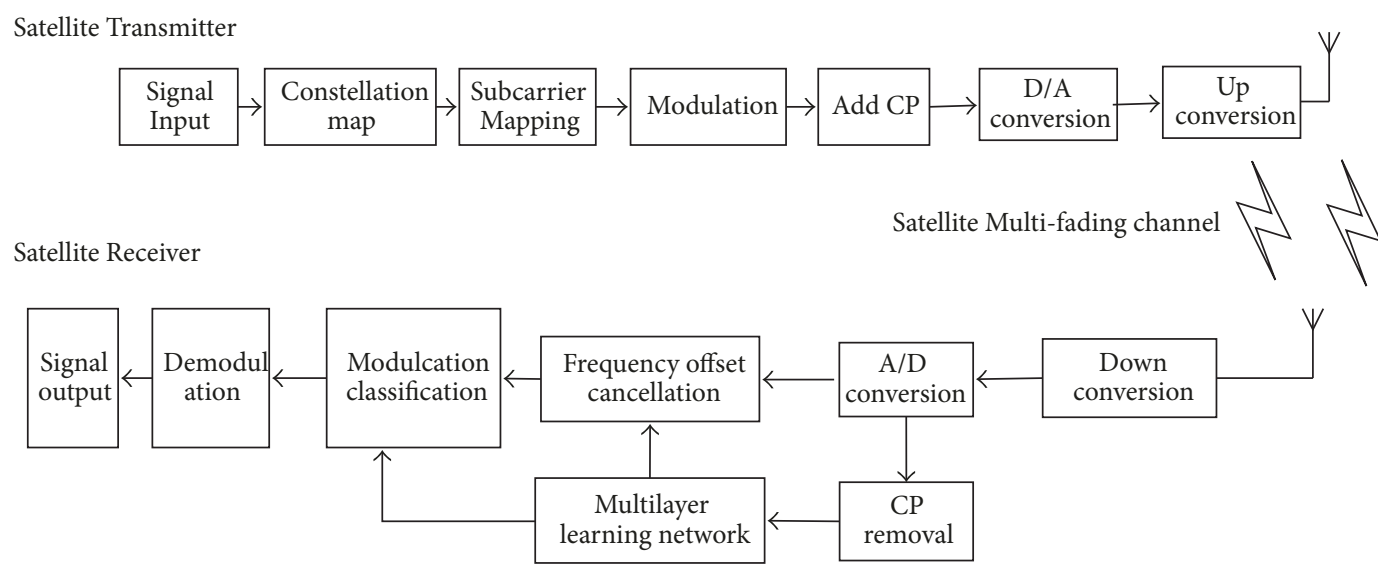

FIGURE 2: Signal processing block diagram in satellite to ground communication link.

firstly, and then signal enters into the frequency offset and modulation classification block based on multilayer learning network. After the corresponding demodulation and decoding, the information can be adopted finally.

\section{Multilayer Learning Network for Modulation Classification Assisted with Frequency Offset Cancellation}

3.1. Motivation of Multilayer Learning Network. The optimization goal of modulation classification for satellite communication signal with frequency offset is the minimum mean square (MSE) between the received signal $r(n)$ with carrier frequency offset and the expected output signal, which could be expressed as $\min \|r(n)-s(n)\|^{2}$.

This equation implicates that the optimization cost function exhibits a strong nonlinear behavior. So it is hard to solve the problem with linear method. Reference [16] also involved a nonlinear regression problem that is also hard to solve. Therefore, in the present work, a nonlinear weight solution method based on multilayer learning network is proposed to solve the nonlinearity problem.

Due to carrier frequency offset interference existing, it seriously influences modulation classification for satellite communication signal. In accordance with the signal processing flow, the proposed multilayer learning network is divided into two parts as shown in Figure 3. The first part is carrier frequency offset cancellation module, and the second part is modulation classification module. Carrier frequency offset module can greatly improve the probability of correct classification for the modulation classification.

Multilayer neural network mechanism for frequency offset interference and modulation mode classification are trained separately.

Due to the formation principle of frequency offset interference in satellite communication system, the required multilayer neural network mechanism for frequency offset interference and modulation mode classification are different. And the weights required by the frequency offset interference cancellation neural network and modulation mode

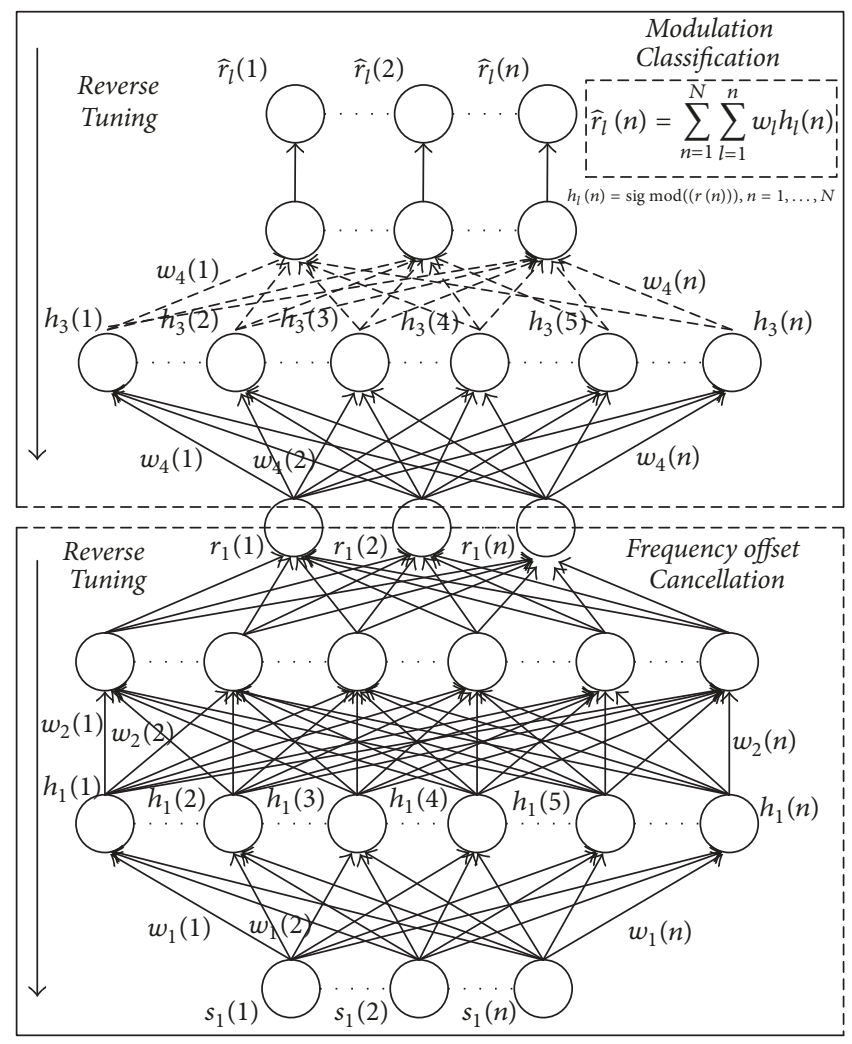

FIGURE 3: Signal processing flow of multilayer learning network for modulation classification of assisted frequency offset cancellation.

classification neural network are greatly different. Therefore, two cascaded neural networks are separately trained and reversely tuned with the same training sequence being used.

3.2. Framework for Communication Signals. Before introducing the multilayer learning network, the framework for communication signal is defined below. Figure 4 shows the defined typical framework, which includes the training symbols and data symbols. The training symbols are known 


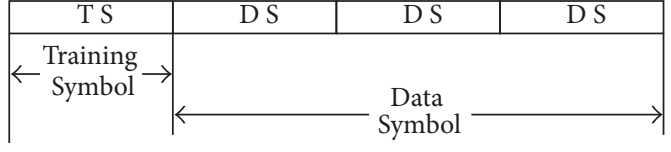

FigURE 4: Framework for communication signals.

by both the transmitter and the receiver, which are also considered as reference symbols. In other words, every training symbol is already known by the receiver. The multilayer learning network can be trained with convergence by training symbols, and the trained network with the optimal weight can be used for frequency offset cancellation and modulation classification.

\section{Multilayer Learning Network for Frequency Offset Cancellation}

4.1. Processing Flow for Carrier Frequency Offset Cancellation. It is effective to obtain optimum weights of multilayer learning network through iterative training symbols. Figure 5 is obtained by $s(n)$ passing through the two-layer learning network with weight $w(n)$, where $s_{1}(n)$ represents the input signal for the first layer learning network and $s_{2}(n)$ represents the input signal for the second layer network. Here, we use two layers for frequency offset cancellation. For current analysis, more layers are not able to obviously improve the performance. The relevant theoretical analysis will be given later. $N$ is defined as the length of symbols for input. $L$ is defined as the training symbols, which can be known by the transmitter and the receiver. After multilayer learning networks are trained adaptively through these symbols, weights will converge. In practice, $L \ll N$.

4.2. First Layer for Carrier Frequency Offset Cancellation. We define cost function as

$$
J(n)=\sum_{n=1}^{N}\|s(n)-r(n)\|^{2}+\lambda^{n} \cdot\|w(n)\|^{2},
$$

where $\lambda$ is exponential weighting factor and is defined as a positive constant value less than unit. $w_{1}(n)$ represents the weight for the first layer learning network and $w_{2}(n)$ represents the weight for the second layer network. $r_{1}(n)$ represents the output for the first layer network and $r_{2}(n)$ represents the output for the second layer network.

We further could obtain through the first layer

$$
J(n)=\sum_{n=1}^{N}\left\|s_{1}(n)-w_{1}^{*}(n) \cdot s_{1}(n)\right\|^{2}+\lambda^{n} \cdot\left\|w_{1}(n)\right\|^{2} .
$$

Optimization goals for weight $w$ can be expressed as

$$
w^{*}=\underset{w \in C^{n \times k}}{\arg \min } J(n) .
$$

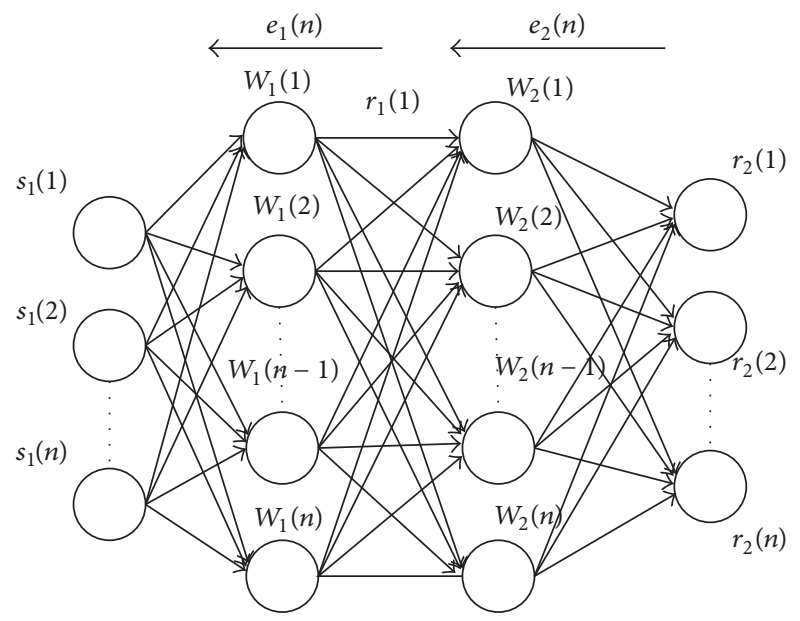

FIGURE 5: Signal processing flow of multilayer learning network for frequency offset cancellation.

$e_{1}(n)$ is defined as the reverse tuning error for the first layer, which is between the $n$th reference symbol and $n$th received symbol. We could obtain

$$
e_{1}(n)=r_{1}(n)-w_{1}^{*}(n) s_{1}(n) .
$$

The gradient vector for cost function with respect to $w_{1}(n)$ can be derived as

$$
\frac{\partial J(n)}{\partial w_{1}(n)}=0 .
$$

After some simple mathematic manipulation, we could obtain

$$
\begin{gathered}
w_{1}(n)=\left[\sum_{n=1}^{N} s_{1}(n) s_{1}^{*}(n)+\lambda^{n} I\right]^{-1} \\
\cdot\left[\sum_{n=1}^{N} s_{1}(n) s_{1}^{*}(n)\right] .
\end{gathered}
$$

Define

$$
H(n)=\sum_{n=1}^{N} s_{1}(n) s_{1}^{*}(n)+\lambda^{n} I .
$$

Note that (9) may be rewritten into the iterative form:

$$
\begin{aligned}
& H(n)=\left[\sum_{n=1}^{N-1} s_{1}(n) s_{1}^{*}(n)+\lambda^{n-1} I\right]+s_{1}(n) s_{1}^{*}(n), \\
& H(n)=\lambda H(n-1)+s_{1}(n) s_{1}^{*}(n) .
\end{aligned}
$$

We could also obtain $w_{1}(n)$ in iterative form:

$$
w_{1}(n+1)=w_{1}(n)+H^{-1}(n) s_{1}(n) e_{1}^{*}(n) .
$$

Substituting $e^{j 2 \pi \xi n / N}$ into (6), we finally get

$$
\begin{aligned}
e_{1}(n) & =s_{1}(n)-r_{1}(n) \\
& =s_{1}(n)-s_{1}(n) \cdot e^{j 2 \pi \xi n / N} \cdot w^{*}(n) \\
& =s_{1}(n) \cdot\left[1-e^{j 2 \pi \xi n / N} \cdot w^{*}(n)\right],
\end{aligned}
$$


where

$$
\begin{aligned}
& s_{1}(n) e^{*}(n)=C \cdot\left[e^{j 2 \pi n \xi / N}-w_{1}(n)\right], \\
& C=s(n) \cdot s^{*}(n), n>1 \\
& H_{1}^{-1}(n)=\left[\sum_{n=1}^{N} s_{1}(n) s_{1}^{*}(n)+\lambda^{n} I\right]^{-1} \\
& =\left[\sum_{n=1}^{N} s_{1}(n) \cdot e^{j 2 \pi n \xi / N} s_{1}^{*}(n) \cdot e^{-j 2 \pi n \xi / N}+\lambda^{n} I\right]^{-1} \\
& =D .
\end{aligned}
$$

After manipulation, we could obtain that $C$ is also a constant value.

Updating $w(n)$, we obtain

$$
\begin{aligned}
w_{1}(n+1)= & w_{1}(n)+C e^{*}(n) \cdot s_{1}(n) \\
= & w_{1}(n)+C D \cdot\left[e^{j 2 \pi n \xi / N}-w_{1}(n)\right] \\
= & (1-C D) w_{1}(n)+C D \cdot e^{j 2 \pi n \xi / N}, \\
w_{1}(n)= & (1-C D)^{n} \cdot w_{1}(0)+C D \cdot(1-C D)^{n-1} \\
& \cdot \sum_{n=1}^{N-1}(1-C D)^{-n} \cdot e^{j 2 \pi n \xi / N}, \quad n>0 .
\end{aligned}
$$

Taking the limit, we could obtain

$$
w_{\infty}=\lim _{n \rightarrow \infty} w(n)=\frac{C D}{e^{j 2 \pi \xi / N}-(1-C D)} e^{j 2 \pi n \xi / N} .
$$

After mathematical operation, the first layer output will be obtained as

$$
\begin{aligned}
r_{1}(n)= & w_{\infty}^{*} \cdot s_{1}(n) \\
= & \frac{C D}{e^{-j 2 \pi \xi / N}-(1-C D)} e^{-j 2 \pi n \xi / N} \cdot x(n) \\
& \cdot e^{j 2 \pi n \xi / N}=\frac{C D}{e^{-j 2 \pi \xi / N}-(1-C D)} \cdot s_{1}(n) \\
= & T \cdot x(n)
\end{aligned}
$$

where

$$
T=\frac{C D}{e^{-j 2 \pi \xi / N}-(1-C D)} .
$$

From (17), we can obtain that the output has the product of $s_{1}(n)$ and $e^{-j 2 \pi n \xi / N}$, and the input symbol $s_{1}(n)$ can be compensated with weights of multilayer learning network. When $\xi \ll 1$, we could obtain that $T \ll 1$. So we have that the interference induced by $F$ could be ignored. If the frequency offset $\xi$ is so large, $F$ would induce the residual frequency offset, which could also destroy the output signal $s_{2}(n)$. So we establish the second layer for residual frequency offset cancellation. $\zeta$ is defined as the residual interference, which is caused by interference part $T$. We could also get this result from constellation simulation as shown in Figure 7. This disturbance also causes the phase rotation of ideal signal, so signal passing through the first layer could be expressed as

$$
s_{2}(n)=x(n) \cdot e^{j 2 \pi \zeta / N}+w(n)
$$

where $\zeta$ is the residual frequency offset; $e^{j 2 \pi \zeta / N}$ could only induce the phase rotation for $s_{2}(n)$.

4.3. Second Layer for Carrier Residual Frequency Offset Cancellation. The second layer for signal processing is similar to the first layer.

Updating $w_{2}(k)$,

$$
\begin{aligned}
w_{2}(k+1) & =w_{2}(k)+B e_{2}^{*}(k) \cdot Y(k) \\
& =w_{2}(k)+B C \cdot\left[e^{j 2 \pi \zeta / N}-w_{2}(k)\right] \\
& =(1-B C) w_{2}(k)+B C \cdot e^{j 2 \pi \zeta / N},
\end{aligned}
$$

where

$$
\begin{aligned}
H_{2}^{-1}(n)=\left[\sum_{n=1}^{N} s_{2}(n) s_{2}^{*}(n)+\lambda^{n} I\right]^{-1} \\
\quad=\left[\sum_{n=1}^{N} s_{2}(n) \cdot e^{j 2 \pi n \xi / N} s_{2}^{*}(n) \cdot e^{-j 2 \pi n \xi / N}+\lambda^{n} I\right]^{-1} \\
\quad=B .
\end{aligned}
$$

After manipulation, we could obtain that $B$ is also a constant value.

$$
\begin{aligned}
w_{2}(n)= & (1-B C)^{n} \cdot W(0)+B C \cdot(1-B C)^{n-1} \\
& \cdot \sum_{n=0}^{N-1}(1-B C)^{-n} \cdot e^{\frac{j 2 \pi \zeta}{N}}, \quad n \geq 0 .
\end{aligned}
$$

Taking the limit, we will obtain

$$
w_{2}=\lim _{n \rightarrow \infty} w(n)=\frac{B C}{1-(1-B C)} e^{j 2 \pi \zeta / N}=e^{j 2 \pi \zeta / N} .
$$

At the high SNR, the noise can be ignored; the output signal will be

$$
r(n)=w_{2}^{*} \cdot s_{2}(n)=e^{-j 2 \pi \zeta / N} \cdot x(n) \cdot e^{j 2 \pi \zeta / N}=x(n) .
$$

From (23), we can obtain that the output has the product of $s_{2}(n)$ and $e^{-j 2 \pi \zeta / N}$; we could get the signal $x(n)$ with non-frequency offset. We therefore conclude that the symbol $s_{2}(n)$ with residual frequency offset can be compensated with second layer of multilayer learning network completely.

The performance for frequency offset cancellation of multilayer learning network could be obtained by the constellation simulation. Consider that SNR is $20 \mathrm{~dB}$ and the number of subcarriers is set as 256. Figure 6 gives out the 


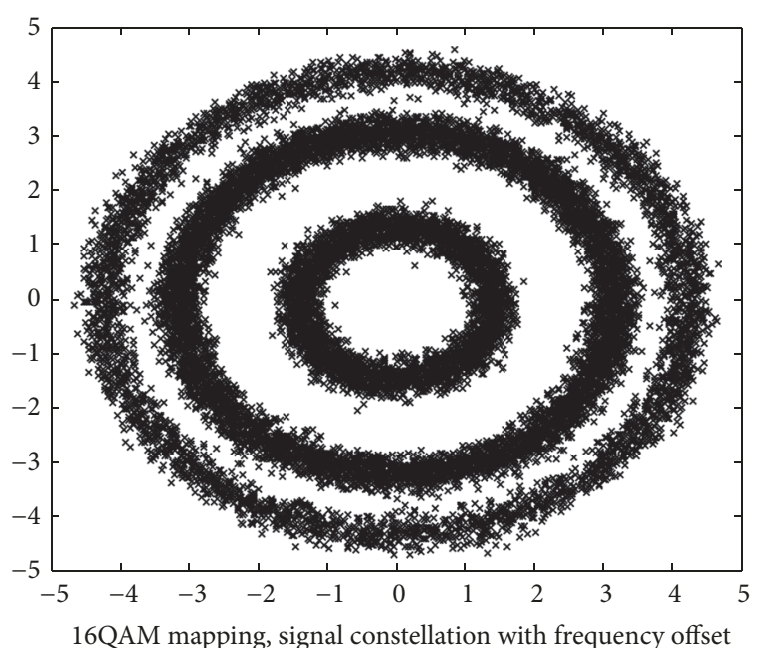

FIGURE 6: Signal constellation with frequency offset $\xi=0.3$.

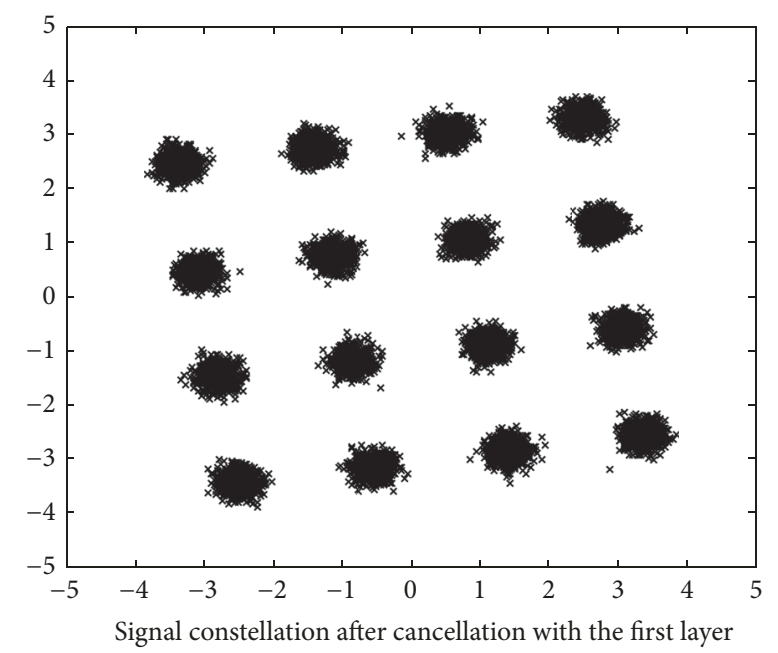

FIGURE 7: Signal constellation after cancellation with first layer of multilayer learning network, with frequency offset $\xi=0.3$.

constellation of base band signal with the frequency offset $\xi=0.3$. Figure 7 presents the constellation after the first layer scheme cancellation. Figure 8 presents the constellation after the second layer scheme cancellation. As obtained from these figures, the first layer scheme may cancel the frequency offset completely when frequency offset value is smaller. But, for a larger frequency offset value, there also exists residual frequency offset. After the second layer, scheme may cancel the frequency offset completely when frequency offset value is larger.

\section{Multilayer Learning Network for Frequency Offset Cancellation}

5.1. Multilayer Learning Network for Modulation Classification. Define $\widehat{r}=[\widehat{r}(1), \widehat{r}(2), \ldots, \widehat{r}(n)]$ as the prediction output for the network. $\widehat{r}(n)$ is defined as the ideal output value for the $n$ th-order network.

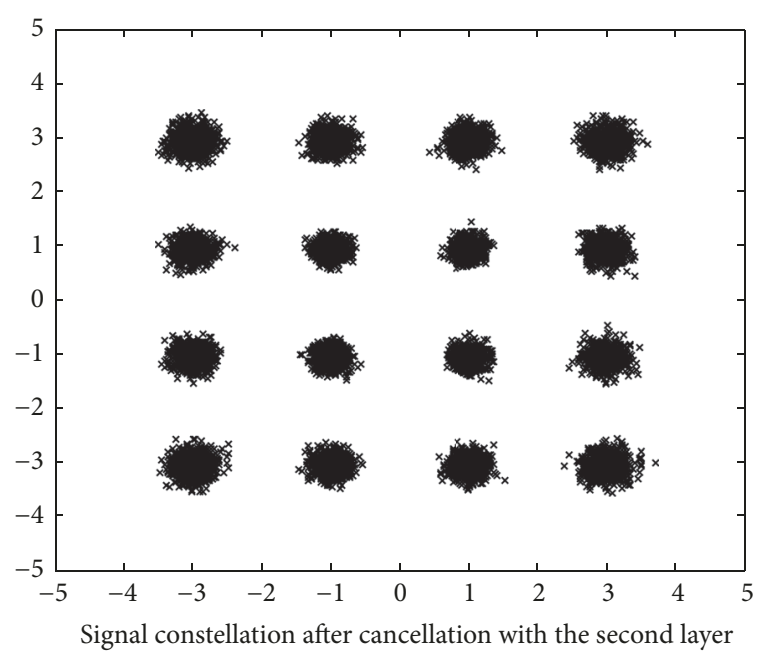

FIGURE 8: Signal constellation after cancellation with second layer of multilayer learning network, with frequency offset $\xi_{i}=0.3$.

The output estimator value of the $n$th layer network can be reconstructed with $l$ th hidden layer unit and the weight $w$; the $l$ th layer network can be expressed as

$$
\widehat{r}_{l}(n)=\sum_{n=1}^{N} \sum_{l=1}^{n} w_{l} h_{l}(n)
$$

where $w_{l}$ is defined as the weight for the $l$ th hidden layer.

$h_{l}$ exists as the $l$ th hidden layer activation function, which is obtained by the following activation function:

$$
\operatorname{sig} \bmod h(x)=\frac{1}{1}+e^{-x}
$$

From the above, we can obtain that the output response can be obtained through $k$ hidden layer unit, which can be expressed as

$$
\widehat{r}_{l}(n)=\sum_{n=1}^{N} \sum_{l=1}^{n} w_{l} h_{l}(n), \quad n=1, \ldots, N
$$

Define $\{(r(1), x(1)),(r(2), x(2)), \ldots,(r(n), x(n))\}, n \ll t$, as training, $x(n)$ is the ideal for the $n$th training unit, and $r(n)$ is the target output of the $n$th training unit.

In order to improve prediction and classification efficiency for the multilayer learning network, the expected forecast direction is through $k$ th hidden layer unit and $l$ th layer network training; signal output $r(n)$ error is $E_{r}=\| r_{l}(n)-$ $\widehat{r}_{l}(n) \|^{2} \rightarrow 0$.

Multilayer network input sequence $r(n)$ is the multipath fading signal. After the $l$ th depth learning network training, the $l$ th hidden layer for unit $h(n)$ can be expressed as

$$
h_{l}(n)=\operatorname{sig} \bmod ((r(n))), \quad n=1, \ldots, N
$$


and expanded as

$$
\begin{aligned}
J(W) & =\sum_{n=1}^{N} \sum_{l=1}^{n}\left\|\left(\widehat{r}_{l}(n)\right)-\left(r_{l}(n)\right)\right\|^{2} \\
& =\sum_{n=1}^{N} \sum_{l=1}^{n}\left(\left(\widehat{r}_{l}(n)\right)-\left(r_{l}(n)\right)\right) \overline{\left(\left(\widehat{r}_{l}(n)\right)-\left(r_{l}(n)\right)\right)} .
\end{aligned}
$$

By substitution, we could obtain

$J(W)$

$$
=\sum_{n=1}^{N} \sum_{l=1}^{n}\left(w_{l} h(n)-r_{l}(n)\right) \overline{\left(\sum_{n=1}^{N} w_{l} h(n)-\left(r_{l}(n)\right)\right)} .
$$

Obtaining $w_{l}$ for the gradient from the above equation, we could obtain

$$
\frac{\partial J(W)}{\partial w_{l}}=\sum_{n=1}^{N} h(n)\left[\sum_{l=1}^{n} \bar{w}_{l} \bar{h}(n)-\left(\bar{r}_{l}(n)\right)\right] .
$$

Then the available is simplified:

$$
\sum_{l=1}^{n} \bar{w}_{l} \sum_{n=1}^{N} h(n) \bar{h}_{l}(t)=\sum_{n=1}^{N}\left(\bar{r}_{l}(n)\right) h(n) .
$$

The amplitude weights can be expressed as the above formula and can be represented by the matrix, which can be simplified:

$$
\bar{W}=M \Omega^{-1}
$$

where

$$
\begin{aligned}
\Omega_{l} & =\sum_{n=1}^{N} h(n) \bar{h}_{l}, \quad l=1, \ldots, n, \\
M_{l} & =\sum_{n=1}^{N} \ln \bar{r}_{l}(n) h(n), \quad l=1, \ldots, n .
\end{aligned}
$$

5.2. Reverse Parameter Adjustment. A parametric model for forward training networks $\{h, w\}$ should be adjusted as reversed parameter. The energy model $T$ is used to obtain the gradient for parameters $\{h, w\}$. Through Contrastive Divergence method, we can obtain

$$
\begin{aligned}
& \frac{\partial J(w)}{\partial w_{i j}}=\langle w\rangle_{p_{0}}-\langle w\rangle_{p_{M}}, \\
& \frac{\partial J(h)}{\partial h_{j}}=\left\langle h_{j}\right\rangle_{p_{0}}-\left\langle h_{j}\right\rangle_{p_{M}},
\end{aligned}
$$

where $\langle\cdot\rangle_{p_{0}}$ indicates the expectation of the data distribution and $\langle\cdot\rangle_{p_{M}}$ indicates the input data after Gibbs sampling. steps:

The parameters $\{h, w\}$ can be adjusted by the following

$$
\begin{aligned}
& w_{l k}^{p}=w_{0}^{p}+\eta \frac{\partial J\left(w^{p-1}\right)}{\partial w^{p}}, \\
& h_{l k}^{p}=h_{0}^{p}+\eta \frac{\partial J\left(h^{p-1}\right)}{\partial h^{p}}
\end{aligned}
$$

where $\eta$ is the learning rate.

\section{Experimental Classification Results and Analysis}

Modulation mode commonly used in satellite communication, QPSK, 8PSK, and QAM are defined as the test sets. These modulation signals are defined as the narrowband signals with white Gaussian noise, which are modulated into the signals with narrowband Gaussian white noise. Signal carrier frequency is defined as $f_{c}=1000 \mathrm{kHz}$, symbol rate is defined as $f_{c}=180 \mathrm{kHz}$, and sampling rate is defined as $f_{s}=4 f_{c}=$ $4000 \mathrm{kHz}$.

The digital signal modulation mode is generated by random generator. Multilayer learning network is simulated by Monte Carlo simulation for 20,000 tests. $\xi_{\max }$ is the maximum normalized frequency offset, which is defined as the ratio between frequency offset and bandwidth.

Typical multipath model is established in Table 1. The main channel adopts the Rice channel model. The secondorder model is Rayleigh channel model.

The measured results for urban environment were proposed in [51] with signal carrier frequency of $1.82 \mathrm{GHz}$. Table 1 shows the measured parameters of the urban environment, rural environment, and suburban environment.

6.1. BER Performance versus SNR. Firstly, we simulate BER versus SNR through frequency offset cancellation performance. The bit-error-rates (BER) versus average SNR for $\xi_{\max }=0.05$ and $\xi_{\max }=0.15$ are shown in Figures 9 and 10, respectively. The results with no frequency offset, no compensation, MMSE equalization algorithm [3], iterative algorithm proposed in [15], and the proposed compensation method are presented. By investigating these figures, it is clearly shown that no cancellation suffers from ICI and has a high error floor. MMSE algorithm performs well, since ICI and MUI can be removed. MMSE-SIC algorithm performs better than MMSE algorithm as the effect of noise enhancement can be significantly reduced. It is noticed that the more precise frequency offset can be obtained by the iterative algorithm at the cost of the complex iterative computation and estimation. However, the two layers of multilayer learning network exhibit the best performance.

6.2. PCC Performance versus SNR at Different Modulation. Secondly, we simulate PCC performance versus SNR. Figure 11 shows the PCC of the multilayer learning network at SNR from $-5 \mathrm{~dB}$ to $20 \mathrm{~dB}$. The frequency offset is defined as $\xi_{\max }=0.05$. Within the same training sequence for each modulation mode, 2048 sampling points constitute a frame. 100 groups are set as training set; that is, $N=100$. The remaining 1900 groups are test set. It can be seen from the figure that when the SNR is greater than $0 \mathrm{~dB}$, the PCC of the multilayer learning network is above $93 \%$ for the above modulation scheme. At SNR greater than $5 \mathrm{~dB}$, the PCC is more than $96 \%$. Therefore, it is obtained from the simulation that the proposed multilayer learning network assisted with frequency offset cancellation has a better PCC for different signal modulation types. 
TABLE 1: Channel model parameter in urban environment.

\begin{tabular}{lccccc}
\hline Tap & $\begin{array}{c}\text { Distribution } \\
\text { function }\end{array}$ & Parameter & $\begin{array}{c}\text { Parameter } \\
\text { distribution }\end{array}$ & $\begin{array}{c}\text { Numerical } \\
\text { value/dB }\end{array}$ & $\begin{array}{c}\text { Time } \\
\text { delay/ns }\end{array}$ \\
\hline 1 & LOS:Rician & Rice factor & $K$ & 5.3 & 0 \\
& nLOS:Rayleigh & Average multipath power & $2 \sigma_{l}^{2}$ & -12.1 & -17.0 \\
2 & Rayleigh & Average multipath power & $2 \sigma_{l}^{2}$ & -18.3 & 60 \\
3 & Rayleigh & Average multipath power & $2 \sigma_{l}^{2}$ & -19.1 & 100 \\
4 & Rayleigh & Average multipath power & $2 \sigma_{l}^{2}$ & -22.1 & 130 \\
5 & Rayleigh & Average multipath power & $2 \sigma_{l}^{2}$ & 250 \\
\hline
\end{tabular}

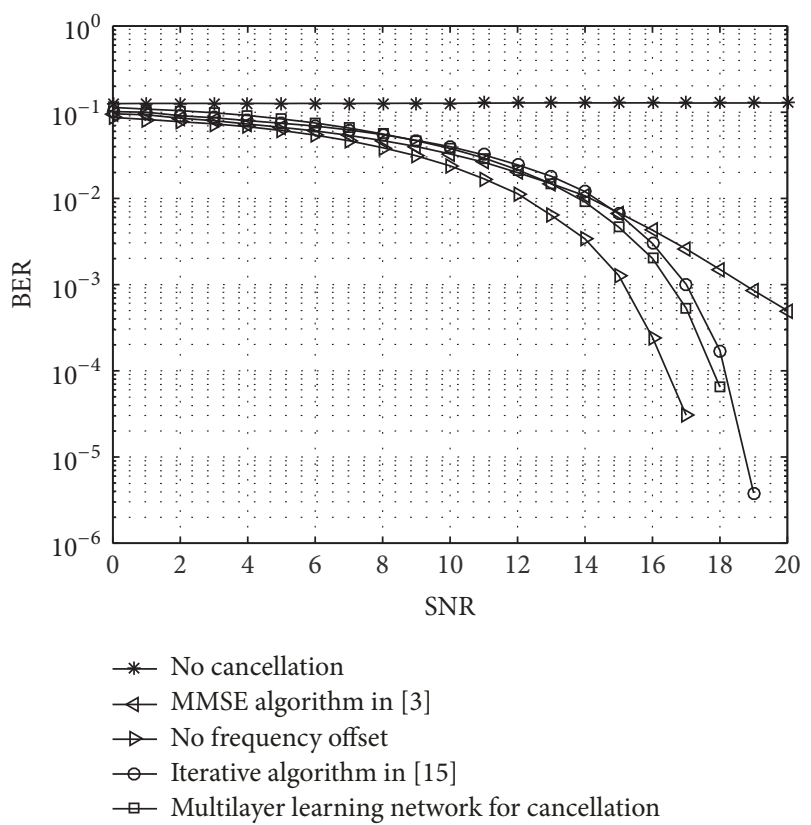

FIGURE 9: BER curves under different average SNR, with frequency offset $\xi_{\max }=0.05$.

6.3. PCC Performance versus SNR at Different Training Length. Figure 12 shows PCC of satellite to ground link signal modulation pattern under different training lengths. Under the different training sequence conditions for each modulation mode, 2048 data frames constitute a group. Frequency offset is defined as $\xi_{\max }=0.05$. For classification modulation within 2000 groups, we choose different lengths of training data, 20 groups, 40 groups, and 60 groups for training.

Through the above method, PCC of multilayer learning network including BPSK, QPSK, and 8PSK modulation signal are tested. It can be seen from Figure 7 that when the training length is greater than 20 groups, the PCC is above $93 \%$ for the above modulation mode. When it is greater than 40 groups, the PCC is more than $96 \%$. Therefore, it is possible to know from the simulation that the proposed multilayer learning network has a better PCC for different types of signal modulation. The proposed multilayer learning network has more profound signal features.

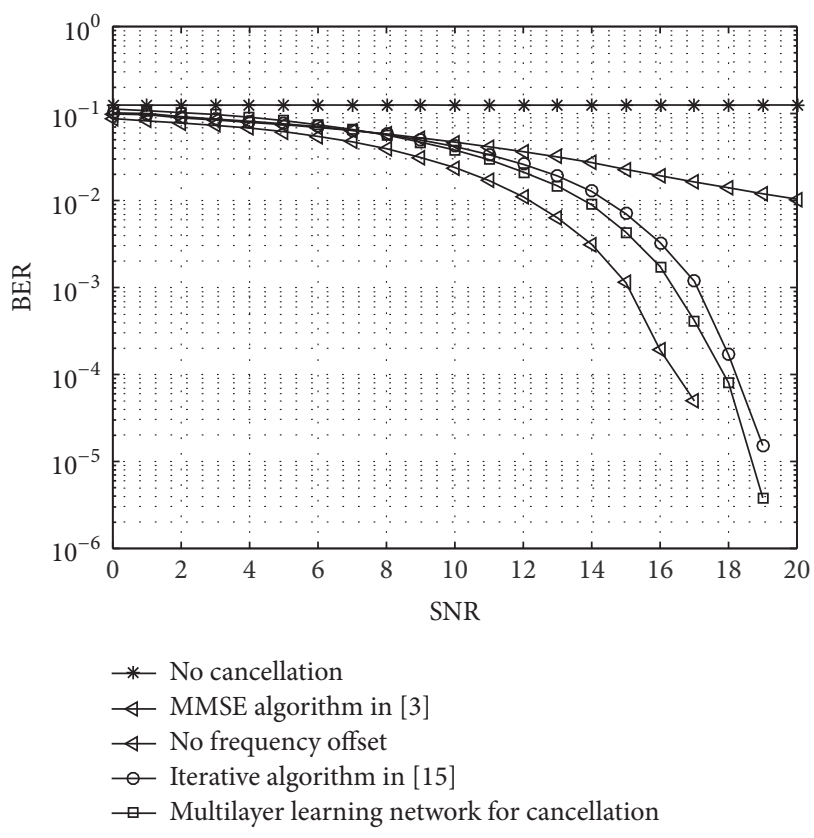

FIGURE 10: BER curves under different average SNR, with frequency offset $\xi_{\max }=0.15$.

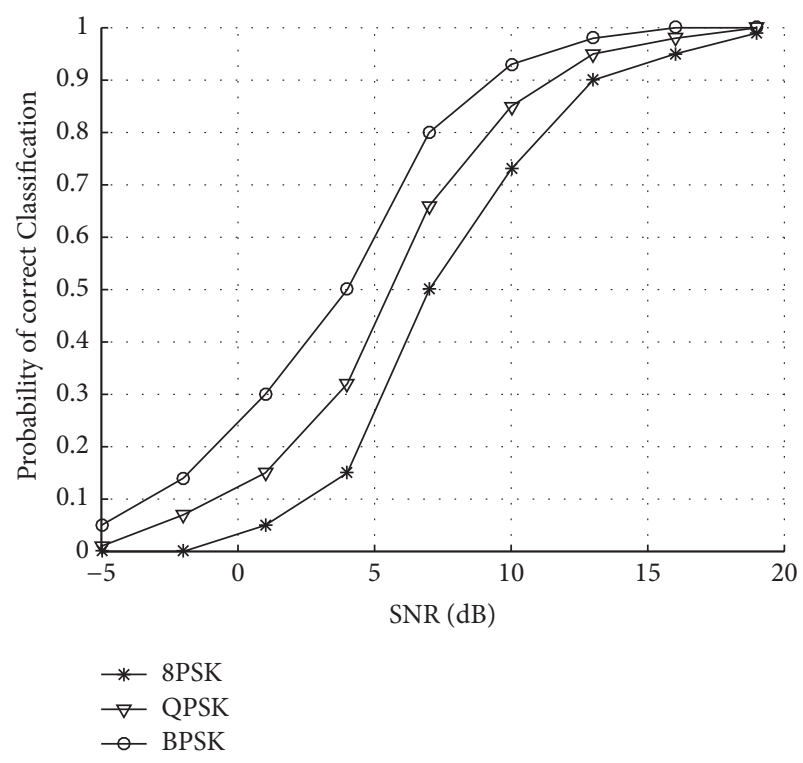

FIGURE 11: Probability of Correct Classification for the proposed learning network based on different modulation. 


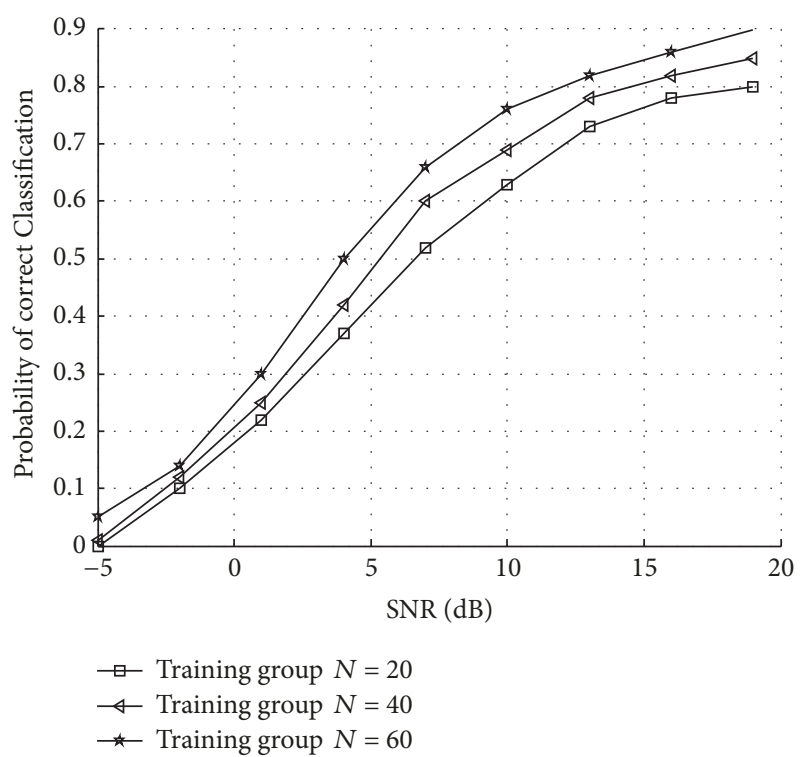

FIgURE 12: Probability of Correct Classification for the proposed learning network based on different lengths of training sequences.

6.4. Probability of Correct Classification Simulation at Different SNR. Figure 13 shows the PCC of proposed multilayer learning network from $-5 \mathrm{~dB}$ to $20 \mathrm{~dB}$ compared with different algorithms proposed in $[20,26,46]$. Define the same training sequence for each modulation mode. 2048 data points constitute a frame, and 1024 frames constitute a group. For 2000 groups, 100 groups are training sets; that is, $N=100$. The remaining 1900 groups are defined as the test group. When SNR $=0 \mathrm{~dB}$, PCC for communication signal modulation mode of all algorithms is below $90 \%$, and the performance of the algorithm is affected by noise interference.

When $\mathrm{SNR}=5 \mathrm{~dB}$, the performance of the algorithm for PCC has increased. The SVM proposed in literature [26] is based on the principle of wavelet packet decomposition criterion, and the PCC performance is higher based on making full use of the wavelet packet features. The PCC of SVM increases rapidly. Therefore, SVM has better PCC in Gaussian white noise. However, in case of frequency offset status, the performance for wavelet packet decomposition is constrained; thus the SVM algorithm does not show an advantage. The modulation classification algorithm based on sixth-order cumulants is proposed in literature [20]; the performance is stable under different SNR conditions. This is because the cumulants features are constrained by the frequency offset status. With frequency offset increasing, cumulants-based signal features are constrained. It can be seen from the simulation results that the proposed algorithm has high accuracy and stability under different SNR conditions. Compared with the KNN algorithm proposed in [46], the proposed multilayer learning has higher PCC under the same SNR condition. This is because the proposed multilayer learning network assisted frequency offset using the multilayer network weights for signal reconstruction.

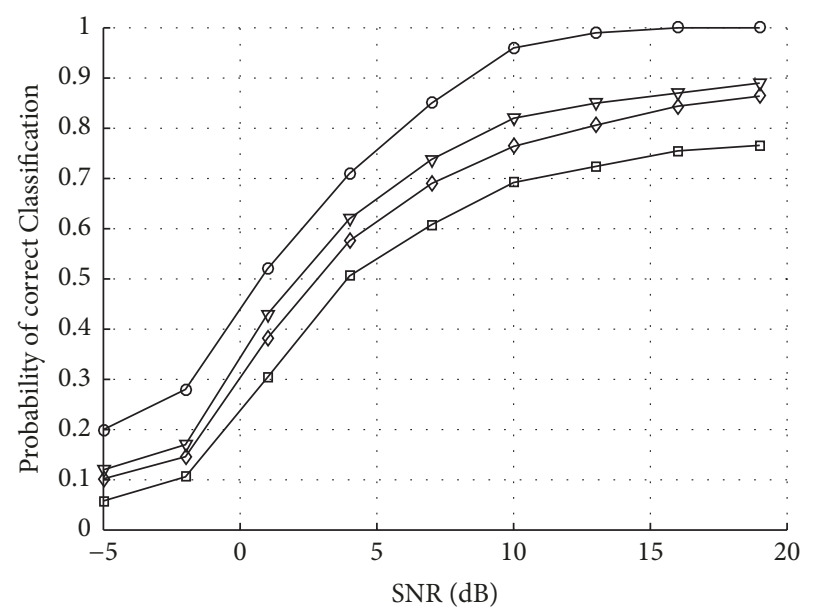

$$
\begin{aligned}
& \multimap \text { Proposed multilayer learning network } \\
& \square \text { KNN network in [46] } \\
& \triangleleft \text { SVM based on wavelet packet in [26] } \\
& \square-\text { Six-order cumulants in [20] }
\end{aligned}
$$

FIgURE 13: Probability of Correct Classification simulation based on different signal-to-noise ratio for different algorithms.

TABLE 2: Performance analysis of different algorithms.

\begin{tabular}{lcccc}
\hline SNR & Proposed network & SVM & KNN network & Cumulants \\
\hline $0 \mathrm{~dB}$ & 0.43 & 0.31 & 0.34 & 0.23 \\
$5 \mathrm{~dB}$ & 0.76 & 0.62 & 0.65 & 0.53 \\
$10 \mathrm{~dB}$ & 0.96 & 0.76 & 0.82 & 0.69 \\
$15 \mathrm{~dB}$ & 0.99 & 0.82 & 0.86 & 0.74 \\
$20 \mathrm{~dB}$ & 0.995 & 0.85 & 0.89 & 0.78 \\
\hline
\end{tabular}

When SNR is not less than $10 \mathrm{~dB}$, the Probability of Correct Classification can reach more than $95 \%$.

Table 2 shows the PCC performance of proposed multilayer learning network from $0 \mathrm{~dB}$ to $20 \mathrm{~dB}$, which is compared with different algorithms proposed in [20,26,46].

Table 3 shows the measured parameters for the proposed multilayer learning network, which is compared with different algorithms proposed in $[20,26,46]$.

6.5. Importance for Multilayer Learning Network with MSE Guidelines. For the frequency offset interference cancellation neural network, the interference is induced by the instantaneous linear exponential multiplicative interference, according to relevant proofs given about formulations (15)-(17), (19), (21), (22), and (23) in Sections 4.2 and 4.3.

The MSE criterion can obtain the optimal interference cancellation performance, and the multiplicative exponential linear interference cancellation can be eliminated. Obtaining few training samples according to MSE criterion, the generated weight network can be trained and the optimal interference cancellation performance can be obtained. So it is effective for adopting the MSE criterion to establish the neural network.

In order to prove the effectiveness of the MSE criterion, Figure 14 shows PCC of satellite to ground link signal 
TABLE 3: Parameters analysis of different algorithms.

\begin{tabular}{lcccc}
\hline Method & Proposed network & SVM & KNN network & Cumulants \\
\hline Parameters & Nonlinear & Nonlinear & Nonlinear & linear \\
\hline
\end{tabular}

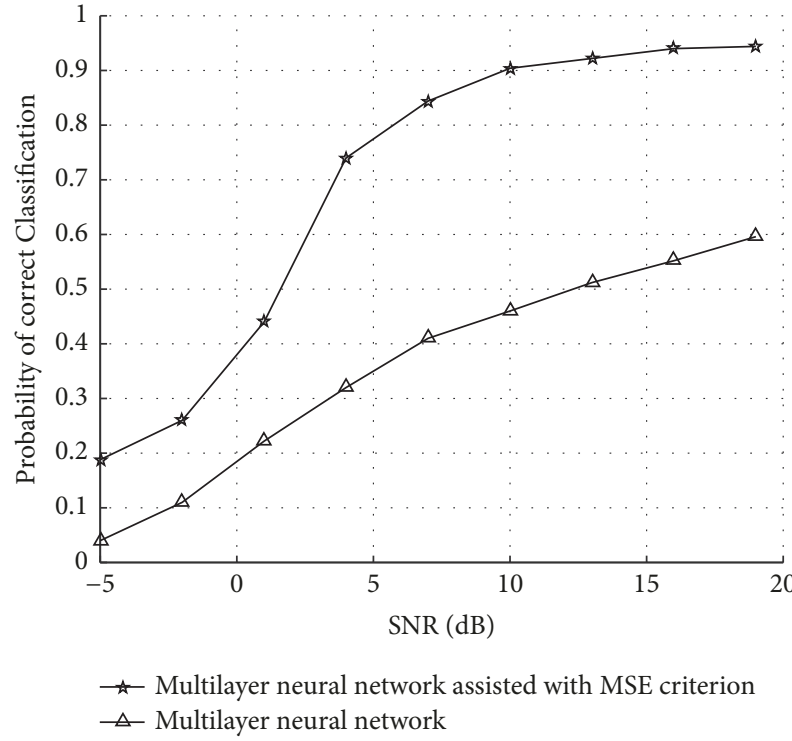

FIGURE 14: Modulation classification assisted with MSE criterion.

modulation classification assisted or not with MSE criterion. For classification modulation within 2000 groups, we choose 200 groups for training. 2048 data frames constitute a group. Frequency offset is defined as $\xi_{\max }=0.05$.

Through the above method, PCC of deep learning network including BPSK, QPSK, and 8PSK modulation signal are tested. It can be seen from Figure 13 that when the training length is the same, the PCC is above $93 \%$ for modulation classification assisted with MSE criterion, which can obtain the optimal interference cancellation performance. Therefore, it is possible to know from the simulation that the proposed deep learning network has a better PCC for different types of signal modulation. Based on MSE criterion, the proposed deep learning network has more profound signal features.

6.6. Overfitting Analysis of Multilayer Learning Assisted with Frequency Offset Cancellation. For the characteristics of satellite link communication signals, the influence of modulation mode classification is mainly due to the interference of larger carrier frequency offset. If the frequency offset interference is not eliminated, more training samples are needed to ensure correct fitting. Due to the innovation of the paper, carrier frequency offset interference cancellation is performed with fewer samples assisted with MSE criterion; thus it improves the accuracy of the modulation mode classification.

Excluding related frequency offset interferences, the classification features are obtained based on the modulation mode, and the model can be established by a small amount of data to ensure the PCC and prevent overfitting of the data.

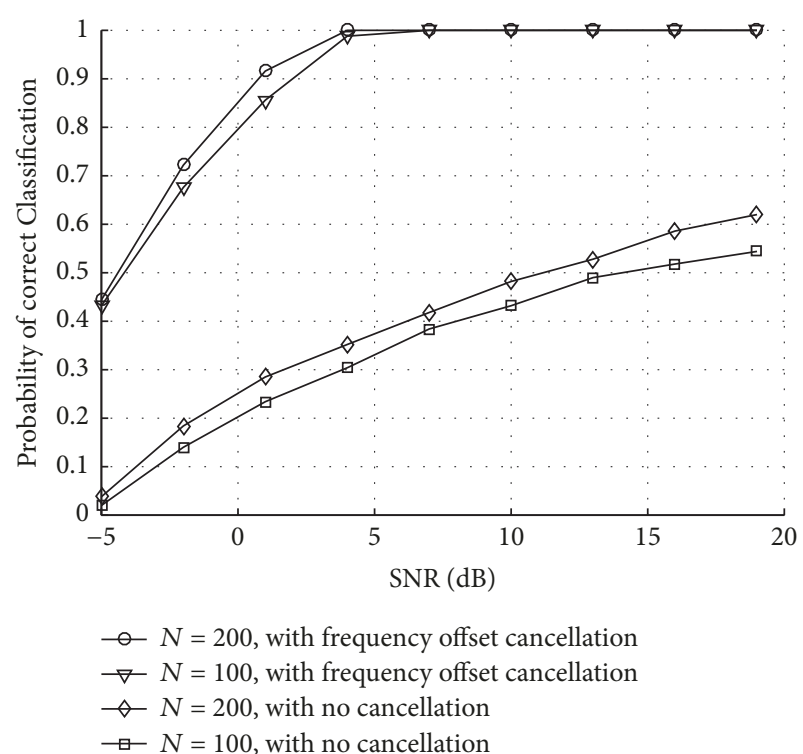

FIGURE 15: PCC for signal modulation classification assisted with frequency offset cancellation.

At the same time, we perform simulation with different training samples. In the condition of frequency offset interference, we could need more training samples in order to ensure a reasonable PCC. And it is the thesis that a small amount of training samples is used to ensure the precancellation of frequency offset interference that makes it possible to obtain a higher classification recognition rate with fewer training sequences.

In order to prove that the small training set can be potentially overfit for the dataset, Figure 15 shows PCC of satellite to ground link signal modulation classification assisted or not with frequency offset cancellation. For classification modulation within 2000 groups, we choose 100 groups and 200 groups for training. 2048 data frames constitute a group. Frequency offset is defined as $\xi_{\max }=0.05$.

Through the above method, PCC of deep learning network including BPSK, QPSK, and 8PSK modulation signal are tested. It can be seen from Figure 12 that when the training length is the small, the PCC is above $97 \%$ for modulation classification assisted with frequency offset cancellation, which can obtain the ideal performance. Therefore, it is possible to know from the simulation that the proposed multilayer learning network has a better PCC for small training set.

\section{Conclusion}

A multilayer learning network assisted with frequency offset cancellation is proposed for modulation classification in satellite to ground link. Larger carrier frequency offset 
greatly degrades the performance of modulation classification. Firstly, we establish cancellation weights for frequency offset through multilayer learning network. Secondly, we establish the weights and hidden layers for modulation classification through multilayer learning network. We also train hidden layers and weight using positive fine-tuning to cancel the interference introduced by frequency offset in the satellite to ground link. Compared with current modulation classification algorithm, the proposed multilayer learning offset greatly improves the PCC. It has been proven that the multilayer learning network model assisted with frequency offset has higher performance for the satellite communication signal classification within the same training sequence.

\section{Conflicts of Interest}

The author declares that there are no conflicts of interest.

\section{Acknowledgments}

This work is supported by the National Natural Science Foundation of China (no. 61501306), the Natural Science Foundation of Liaoning Province of China (no. 2015020026), and the Natural Science Foundation of Liaoning Provincial Department of Education (no. L2015402).

\section{References}

[1] S. Jajodia, P. Ammann, and C. D. McCollum, "Surviving information warfare attacks," The Computer Journal, vol. 32, no. 4, pp. 57-63, 1999.

[2] X. Li, J. Luo, and Y. Cao, "Multi-agent model of information warfare system," in Proceedings of the International Symposium on Communications and Information Technologies, ISCIT '05, pp. 554-557, October 2005.

[3] T. A. Lipinski, "Information warfare, American style," IEEE Technology and Society Magazine, vol. 18, no. 1, pp. 10-19, 1999.

[4] A. Tonello and S. Pupolin, "Performance of single user detectors in multitone multiple access asynchronous communications," in Proceedings of the Vehicular Technology Conference. IEEE 55th Vehicular Technology Conference. VTC Spring '02, pp. 199-203, Birmingham, AL, USA.

[5] C. Z. Ren, U. Tureli, and Y. Yu-Dong, "Analysis of two receiver schemes for interleaved OFDMA uplink," in Proceedings of the Signals, Systems and Computers, Conference Record of the ThirtySixth Asilomar Conference, vol. 2, pp. 1818-1821.

[6] M. Huang, X. Chen, S. Zhou, and J. Wang, "Iterative ICI cancellation algorithm for uplink OFDMA system with carrierfrequency offset," in Proceedings of the 62nd Vehicular Technology Conference, VTC 2005, pp. 1613-1617, USA, September 2005.

[7] D. Huang and K. B. Letaief, "An interference-cancellation scheme for carrier frequency offsets correction in OFDMA systems," IEEE Transactions on Communications, vol. 53, no. 7, pp. 1155-1165, 2005.

[8] J. Choi, C. Lee, H. W. Jung, and Y. H. Lee, "Carrier frequency offset compensation for uplink of OFDM-FDMA systems," IEEE Communications Letters, vol. 4, no. 12, pp. 414-416, 2000.

[9] M.-O. Pun, M. Morelli, and C.-C. J. Kuo, "Iterative detection and frequency synchronization for OFDMA uplink transmissions,"
IEEE Transactions on Wireless Communications, vol. 6, no. 2, pp. 629-639, 2007.

[10] R. Fantacci, D. Marabissi, and S. Papini, "Multiuser interference cancellation receivers for ofdma uplink communications with carrier frequency offset," in Proceedings of the GLOBECOM '04 - IEEE Global Telecommunications Conference, vol. 5, pp. 28082812, IEEE, 2004.

[11] V. D. Trajković, P. B. Rapajic, and R. A. Kennedy, "Adaptive ordering for imperfect successive decision feedback multiuser detection," IEEE Transactions on Communications, vol. 56, no. 2, pp. 173-176, 2008.

[12] S. Hou and C. C. Ko, "Intercarrier interference suppression for OFDMA uplink in time and frequency selective Rayleigh fading channels," in Proceedings of the 2008 IEEE 67th Vehicular Technology Conference-Spring, VTC, pp. 1438-1442, Singapore, May 2008.

[13] D. Sreedhar and A. Chockalingam, "MMSE receiver for multiuser interference cancellation in uplink OFDMA," in Proceedings of the 2006 IEEE 63rd Vehicular Technology Conference, VTC 2006-Spring, vol. 5, pp. 2125-2129, July 2006.

[14] D. Marabissi, R. Fantacci, and S. Papini, "Robust multiuser interference cancellation for OFDM systems with frequency offset," IEEE Transactions on Wireless Communications, vol. 5, no. 11, pp. 3068-3076, 2006.

[15] K. Raghunath and A. Chockalingam, "SIR analysis and interference cancellation in uplink OFDMA with large carrier frequency and timing offsets," in Proceedings of the IEEE Wireless Communications and Networking Conference, WCNC '07, pp. 996-1001, March 2007.

[16] V. Orlić and M. L. Dukić, "Algorithm for automatic modulation classification in multipath channel based on sixth-order cumulants," in Proceedings of the 9th International Conference on Telecommunication in Modern Satellite, Cable, and Broadcasting Services (TELSIKS '09), pp. 423-426, IEEE, Niš, Serbia, October 2009.

[17] A. Swami and B. M. Sadler, "Hierarchical digital modulation classification using cumulants," IEEE Transactions on Communications, vol. 48, no. 3, pp. 416-429, 2000.

[18] O. Dobre, Y. Bar-Ness, and W. Su, "Robust QAM modulation classification algorithm using cyclic cumulants," in Proceedings of the IEEE Wireless Communications and Networking Conference, pp. 745-748, March 2004.

[19] A. E. El-Mahdy, "Classification of MFSK signals over timevarying flat correlated fading channels under class-A impulsive noise environment," The Journal of the Institution of Electrical Engineers, vol. 151, no. 6, pp. 619-626, 2004.

[20] P. Li, F. Wang, and Z. Wang, "Algorithm for modulation recognition based on high-order cumulants and subspace decomposition," in Proceedings of the 8th International Conference on Signal Processing, ICSP '06, 2006.

[21] M. R. Mirarab and M. A. Sobhani, "Robust modulation classification for PSK/QAM/ASK using higher-order cumulants," in Proceedings of the 6 th International Conference on Information, Communications and Signal Processing, ICICS '07, 2007.

[22] H. You and D.-H. Wang, "Dynamics of multiple-choice decision making," Neural Computation, vol. 25, no. 8, pp. 2108-2145, 2013.

[23] T. Wimalajeewa and P. K. Varshney, "Asymptotic performance of categorical decision making with random thresholds," IEEE Signal Processing Letters, vol. 21, no. 8, pp. 994-997, 2014. 
[24] Y. T. Chan and L. G. Gadbois, "Identification of the modulation type of a signal," Signal Processing, vol. 16, no. 2, pp. 149-154, 1989.

[25] M. L. D. Wong and A. K. Nandi, "Automatic digital modulation recognition using spectral and statistical features with multilayer perceptrons," in Proceedings of the 6th International Symposium on Signal Processing and Its Applications, ISSPA '01, pp. 390-393, August 2001.

[26] F. Zhao, Y. Hu, and S. Hao, "Classification using wavelet packet decomposition and support vector machine for digital modulations," Journal of Systems Engineering and Electronics, vol. 19, no. 5, pp. 914-918, 2008.

[27] N. Ghani and R. Lamontagne, "Neural networks applied to the classification of spectral features for automatic modulation recognition," in Proceedings of the MILCOM '93 - IEEE Military Communications Conference, pp. 111-115, Boston, MA, USA.

[28] M. Lu, X. Xiao, and L. Li, "Cyclic spectral features based modulation recognition," in Proceedings of the 1996 International Conference on Communication Technology Proceedings, ICCT'96. Part 2 (of 2), pp. 792-795, May 1996.

[29] N. P. Ta, "A wavelet packet approach to radio signal classification," in Proceedings of the IEEE-SP International Symposium on Time-Frequency and Time-Scale Analysis, pp. 508-511, 1994.

[30] K. C. Ho, W. Prokopiw, and Y. T. Chan, "Modulation identification of digital signals by the wavelet transform," IEE Proceedings-Radar, Sonar and Navigation, vol. 147, no. 4, pp. 169-176, 2000.

[31] S. Huang, Y. Yao, Y. Xiao, and Z. Feng, "Cumulant based maximum likelihood classification for overlapped signals," IEEE Electronics Letters, vol. 52, no. 21, pp. 1761-1763, 2016.

[32] Y. Yang and S. S. Soliman, "A suboptimal algorithm for modulation classification," IEEE Transactions on Aerospace and Electronic Systems, vol. 33, no. 1, pp. 38-45, 1997.

[33] C. Schreyoegg and J. Reichert, "Modulation classification of QAM schemes using the DFT of phase histogram combined with modulus information," in Proceedings of the Military Communications Conference Proceedings, MILCOM '97, vol. 3, pp. 1372-1376, IEEE, 1997.

[34] B. F. Beidas and C. L. Weber, "Asynchronous classification of mfsk signals using the higher order correlation domain," IEEE Transactions on Communications, vol. 46, no. 4, pp. 480-493, 1998.

[35] D. Boiteau and C. Le Martret, "A general maximum likelihood framework for modulation classification," in Proceedings of the 23rd IEEE International Conference on Acoustics, Speech and Signal Processing, ICASSP '98, pp. 2165-2168, 1998.

[36] A. Ahmad and G. Brown, "Random projection random discretization ensembles - Ensembles of linear multivariate decision trees," IEEE Transactions on Knowledge and Data Engineering, vol. 26, no. 5, pp. 1225-1239, 2014.

[37] M. Biswal and P. K. Dash, "Measurement and classification of simultaneous power signal patterns with an s-transform variant and fuzzy decision tree," IEEE Transactions on Industrial Informatics, vol. 9, no. 4, pp. 1819-1827, 2013.

[38] H. G. Zhang, Z. S. Wang, and D. R. Liu, "A comprehensive review of stability analysis of continuous-time recurrent neural networks," IEEE Transactions on Neural Networks and Learning Systems, vol. 25, no. 7, pp. 1229-1262, 2014.

[39] J. Wang, C.-H. Wang, and C. L. P. Chen, "The bounded capacity of fuzzy neural networks (FNNs) via a new fully connected neural fuzzy inference system (F-CONFIS) with its applications," IEEE Transactions on Fuzzy Systems, vol. 22, no. 6, pp. 1373-1386, 2014.

[40] Z. Lu, J. Sun, and K. Butts, "Multiscale asymmetric orthogonal wavelet kernel for linear programming support vector learning and nonlinear dynamic systems identification," IEEE Transactions on Cybernetics, vol. 44, no. 5, pp. 712-724, 2014.

[41] Z. Hao, L. He, B. Chen, and X. Yang, "A linear support higherorder tensor machine for classification," IEEE Transactions on Image Processing, vol. 22, no. 7, pp. 2911-2920, 2013.

[42] Y. Zhao, C. K. Li, and Z. Wu, "An efficient parallel decision algorithm for recognition of modulation systems in a software radio," IEICE Transactions on Communications, vol. E87-B, no. 13, pp. 174-178, 2004.

[43] A. Swami and B. Sadler, "Modulation classification via hierarchical agglomerative cluster analysis," in Proceedings of the 1st IEEE Signal Processing Workshop on Signal Processing Advances in Wireless Communications, SPAWC'97, pp. 141-144, April 1997.

[44] M. L. D. Wong and A. K. Nandi, "Automatic digital modulation recognition using artificial neural network and genetic algorithm," Signal Processing, vol. 84, no. 2, pp. 351-365, 2004.

[45] P. Urriza, E. Rebeiz, and D. Cabric, "Optimal discriminant functions based on sampled distribution distance for modulation classification," IEEE Communications Letters, vol. 17, no. 10, pp. 1885-1888, 2013.

[46] M. W. Aslam, Z. Zhu, and A. K. Nandi, "Automatic modulation classification using combination of genetic programming and KNN," IEEE Transactions on Wireless Communications, vol. 11, no. 8, pp. 2742-2750, 2012.

[47] J. F. Ossanna, "A model for mobile radio fading due to building reflections: theoretical and experimental fading waveform power spectra," Bell System Technical Journal, vol. 43, no. 6, pp. 2935-2971, 1964.

[48] R. H. Clarke, "A statistical theory of mobile-radio reception," Bell Labs Technical Journal, vol. 47, no. 2, pp. 957-1000, 1968.

[49] C. Loo, "A statistical model for a land mobile satellite link," IEEE Transactions on Vehicular Technology, vol. 34, no. 3, pp. 122-127, 1985.

[50] A. Abdi, W. C. Lau, M. Alouini, and M. Kaveh, "A new simple model for land mobile satellite channels: first-and second-order statistics," IEEE Transactions on Wireless Communications, vol. 2, no. 3, pp. 519-528, 2003.

[51] E. Lutz, M. Werner, and A. Jahn, Satellite Systems for Personal and Broadband Communication Germany, Springer, Berlin, Germany, 2000. 


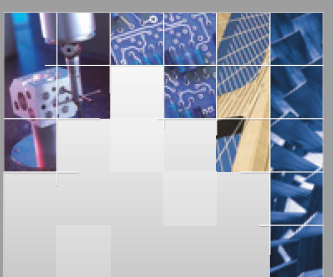

\section{Enfincering}
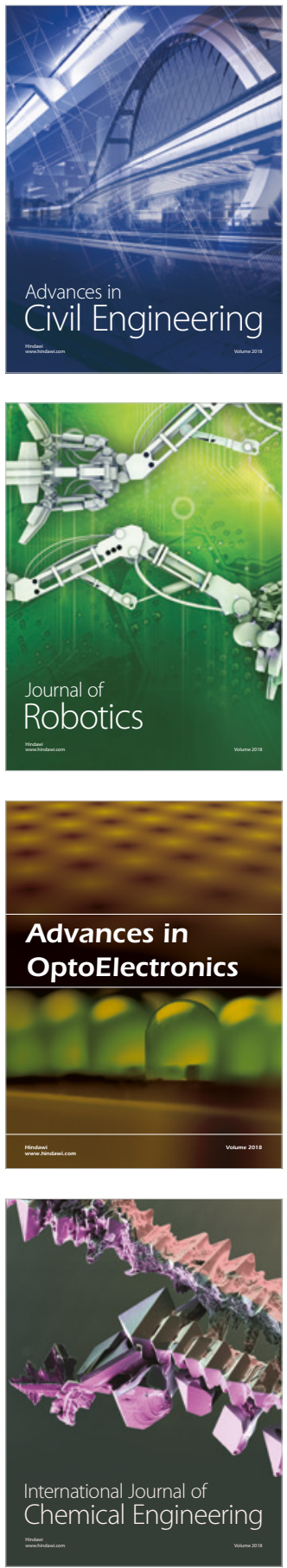

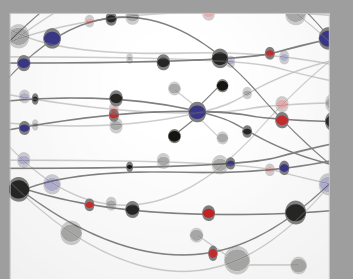

\section{Rotating \\ Machinery}

The Scientific World Journal

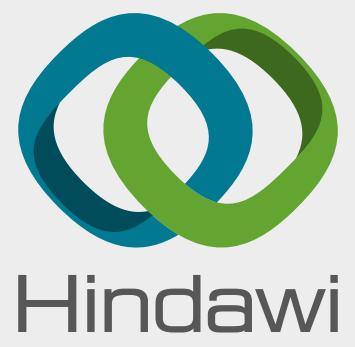

Submit your manuscripts at

www.hindawi.com
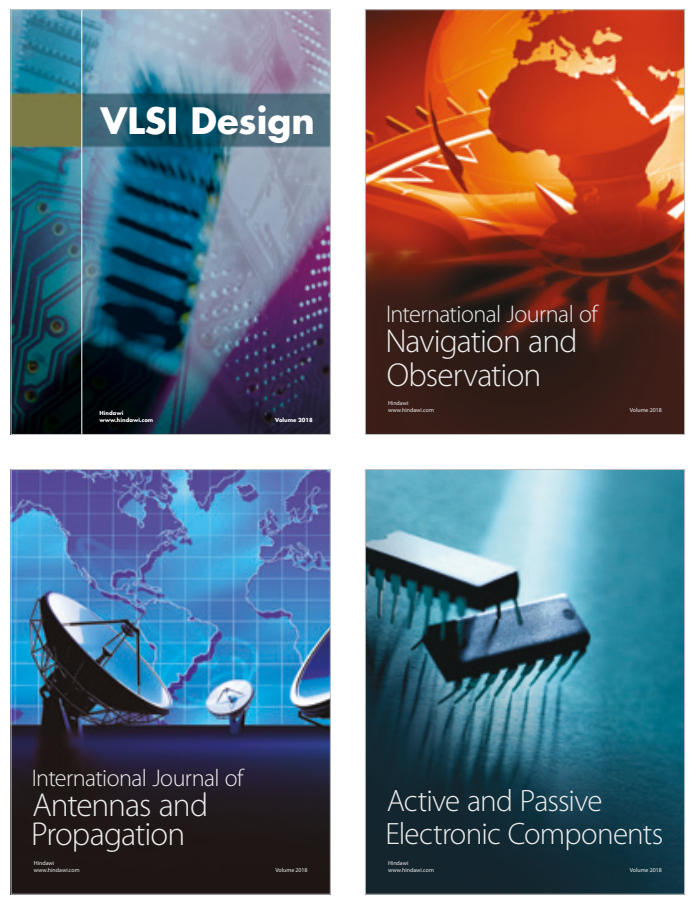
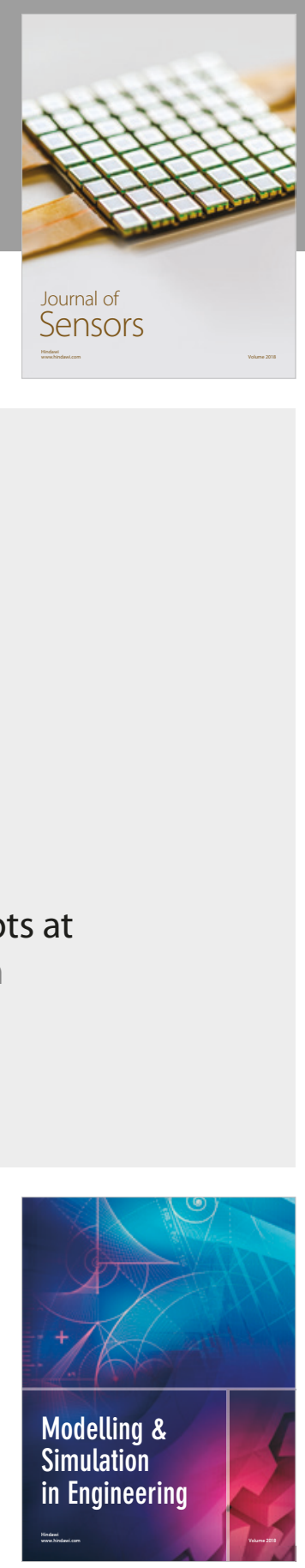

\section{Advances \\ Multimedia}
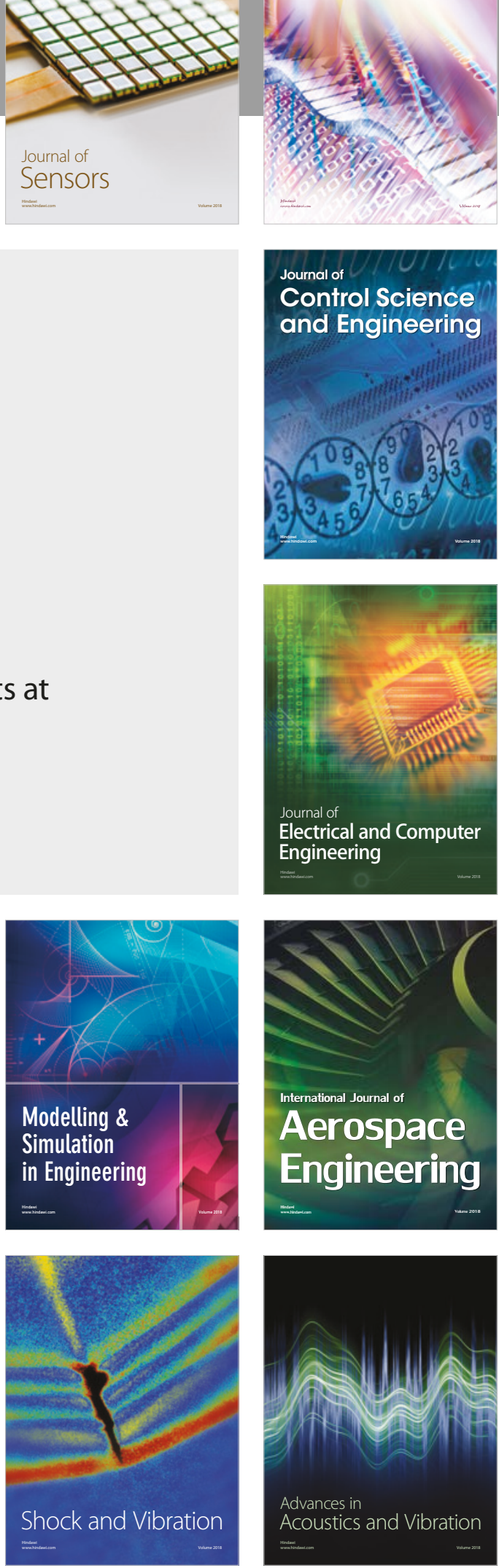